\title{
Vascular Nursing Experience, Practice Knowledge, and Beliefs: Results From the Michigan PICC1 Survey
}

\author{
Vineet Chopra, MD, MSc ${ }^{1,2 *}$, Latoya Kuhn, $\mathrm{MPH}^{2}$, David Ratz, $\mathrm{MS}^{2}$, Scott A. Flanders, MD¹, Sarah L. Krein, PhD, RN¹,2
}

\begin{abstract}
${ }^{1}$ The Division of General Medicine, University of Michigan Health System, Ann Arbor, Michigan; ${ }^{2}$ Patient Safety Enhancement Program and Center for Clinical Management Research, Veterans Affairs Ann Arbor Healthcare System, Ann Arbor, Michigan.
\end{abstract}

BACKGROUND: Peripherally inserted central catheters (PICCs) are increasingly used in hospitalized patients. Yet, little is known about the vascular access nurses who often place them.

METHODS: We conducted a Web-based survey to assess vascular access nursing experience, practice, knowledge, and beliefs related to PICC insertion and care in 47 Michigan hospitals.

RESULTS: The response rate was $81 \%$ (172 received invitations, 140 completed the survey). More than half of all respondents $(58 \%)$ reported placing PICCs for $\geq 5$ years, and $23 \%$ had obtained dedicated vascular access certification. The most common reported indications for PICC insertion included intravenous antibiotics, difficult venous access, and chemotherapy. Many respondents (46\%) reported placing a PICC in a patient receiving dialysis; however, $91 \%$ of these respondents reported receiving approval from nephrology prior to insertion. Almost all respondents (91\%) used ultrasound to find a suitable vein for PICC insertion, and $76 \%$ used electrocardiography guidance to place PICCs. PICC occlusion was reported as the most frequently encountered complication, followed by device migration and deep vein thrombosis. Although $94 \%$ of respondents noted that their hospitals tracked the number of PICCs placed, only $40 \%$ reported tracking duration of PICC use. Relatedly, 30\% of nurses reported that their hospitals had a written policy to evaluate PICC necessity or appropriateness.

CONCLUSION: This survey of vascular nursing experiences highlights opportunities to improve practices such as avoiding PICC use in dialysis, better tracking of PICC dwell times, and necessity. Hospitalists may use these data to inform clinical practice, appropriateness, and safety of PICCs in hospitalized patients. Journal of Hospital Medicine 2016;11:269-275. 2015 Society of Hospital Medicine
Peripherally inserted central catheters (PICCs) are among the most prevalent of venous access devices in hospitalized patients. ${ }^{1,2}$ Although growing use of these devices reflects clinical advantages, such as a reduced risk of complications during insertion and durable venous access, use of PICCs is also likely related to the growth of vascular access nursing. ${ }^{3,4}$ A relatively new specialty, vascular access nurses obtain, maintain, and manage venous access in hospitalized patients. ${ }^{4,5}$ Depending on their scope of practice, these professionals are responsible not only for insertion of devices, such as peripheral intravenous catheters and PICCs, but also nontunneled central venous catheters and arterial catheters in some settings. ${ }^{6}$

Although a growing number of US hospitals have introduced vascular nursing teams, ${ }^{7}$ little is known about the experience, practice, knowledge, and beliefs of vascular access nurses. This knowledge gap is relevant

*Address for correspondence and reprint requests: Vineet Chopra, MD, 2800 Plymouth Road, Building 16 \#432W, Ann Arbor, Ml 48109; Telephone: 734-647-1599; Fax: 734-936-8944; E-mail: vineetc@umich.edu

Additional Supporting Information may be found in the online version of this article.

Published 2015. This article is a US Government work and, as such, is in the public domain of the United States of America.

Received: September 22, 2015; Revised: October 28, 2015; Accepted: November 8, 2015

2015 Society of Hospital Medicine DOI 10.1002/jhm.2523

Published online in Wiley Online Library (Wileyonlinelibrary.com). for hospitalists and hospital medicine as (1) vascular access nurses increasingly represent a key partner in the care of hospitalized patients; (2) the knowledge and practice of these individuals directly affects patient safety and clinical outcomes; and (3) understanding experience, practice, and beliefs of these specialists can help inform decision making and quality-improvement efforts related to PICCs. As hospitalists increasingly order the placement of and care for patients with PICCs, they are also well suited to improve PICC practice.

Therefore, we conducted a survey of vascular access nurses employed by hospitals that participate in the Michigan Hospital Medicine Safety (HMS) Consortium, a Blue Cross Blue Shield of Michigan-funded collaborative quality initiative. ${ }^{6}$ We aimed to understand experience, practice, knowledge, and beliefs related to PICC care and use.

\section{METHODS}

\section{Study Setting and Participants}

To quantify vascular nursing experience, practice, knowledge, and beliefs, we conducted a Web-based survey of vascular nurses across 47 Michigan hospitals that participate in HMS. A statewide quality-improvement initiative, HMS aims to prevent adverse events in hospitalized medical patients through the creation of a data registry and sharing of best practices. The setting and design of this multicenter initiative have been previously described. ${ }^{8,9}$ Although participation is voluntary, each hospital receives payment for participating in the consortium and 
for data collection. Because HMS has an ongoing initiative aimed at identifying and preventing PICC-related complications, this study was particularly relevant for participating hospitals and nurses.

Each HMS site has a designated quality-improvement lead, physician champion, and data abstractor. To coordinate distribution and dissemination of the survey, we contacted the quality-improvement leads at each site and enquired whether their hospital employed vascular access nurses who placed PICCs. Because we were only interested in responses from vascular access nurses, HMS hospitals that did not have these providers or stated PICCs were placed by other specialists (eg, interventional radiology) were excluded. At eligible sites, we obtained the total number of vascular nurses employed so as to determine the number of eligible respondents. In this manner, a purposeful sample of vascular nurses at participating HMS hospitals was constituted.

Participation in the survey was solicited through hospital quality leads that either distributed an electronic survey link to vascular nurses at their facilities or sent us individual email addresses to contact them directly. A cover letter explaining the rationale and the purpose of the survey along with the survey link was then sent to respondents through either of these routes. The survey was administered at all HMS sites contemporaneously and kept open for a period of 5 weeks. During the 5 -week period, 2 e-mail reminders were sent to encourage participation. As a token of appreciation, a \$10 Amazon gift card was offered to those who took the survey.

\section{Development and Validation of the Survey}

We developed the survey instrument (which we call PICC1 as we hope to administer longitudinally to track changes over time) by first conducting a literature search to identify relevant evidence-based guidelines and studies regarding vascular access nursing practices and experiences. ${ }^{10-13}$ In addition, we consulted and involved national and international leaders in vascular access nursing to ensure validity and representativeness of the questions posed. We were specifically interested in nursing background, hospital practices, types of PICCs used, use of various technologies, relationships with healthcare providers, and management of complications. To understand participant characteristics and quantify potential variation in responses, we collected basic participant data including demographics, years in practice, number of PICCs placed, leadership roles, and vascular access certification status. Based on clinical reasoning and existing studies, ${ }^{14,15}$ we hypothesized that responses regarding certain practices (ultrasound use, electrocardiography [ECG] guidance system use), management of complications, or perceptions regarding leadership might vary based on years of experience, number of PICCs placed, or certification status. We therefore examined these associations as prespecified subgroup analyses.
The initial survey instrument was pilot tested with vascular nurses outside of the sampling frame. Based on feedback from the pilot testers, the instrument was refined and edited to improve clarity of the questions. In addition, specific skip patterns and logic were programmed into the final survey to reduce respondent burden and allow participants to seamlessly bypass questions that were contingent on a prior response (eg, use of ECG to place PICCs would lead to a series of questions about ECG-assisted placement only for those respondents who used the technology). This final version of the survey was tested by members of the study team (V.C., L.K., S.L.K.) and then posted to SurveyMonkey for dissemination.

\section{Statistical Analysis}

Descriptive statistics (percentage, $\mathrm{n} / \mathrm{N}$ ) were used to tabulate results. In accordance with our a priori hypothesis that variation to responses might be associated with respondent characteristics, responses to questions regarding insertion practice (eg, use of ultrasound, measurement of catheter:vein ratio, trimming of catheters) and approach to complications (eg, catheter occlusion, deep vein thrombosis [DVT] notification, and PICC removal in the setting of fever) were compared by respondent years in practice (dichotomized to $<5$ vs $>5$ years), volume of PICCs placed ( $<999$ vs $\geq 1000$ ), and certification status (yes/no). Bivariate comparisons were made using $\chi^{2}$ or Fisher exact tests based on the number of responses in a cell as appropriate; 2 -sided $\alpha$ with a $P$ value $<0.05$ was considered statistically significant. All statistical analyses were conducted using SAS version 9.3 (SAS Institute, Cary, NC).

\section{Ethical and Regulatory Oversight}

Because our study sought to describe existing practice without collecting any individual or facility level identifiable information, the project received a "Not Regulated" status by the University of Michigan Medical School Institutional Review Board (HUM00088351).

\section{RESULTS}

Of 172 vascular nurses who received invitations, 140 completed the survey for a response rate of $81 \%$. Respondents reported working in not-for-profit hospitals $(36 \%)$, academic medical centers $(29 \%)$, and forprofit hospitals $(21 \%)$. Although multiple providers (eg, interventional radiology staff and providers, physicians) placed PICCs, 95\% of those surveyed reported that they placed the majority of the PICCs at their institutions. Although most respondents placed PICCs in adult patients $(86 \%)$, a few also placed PICCs in pediatric populations $(17 \%)$. Vascular nursing programs were largely housed in their own department, but some reported to general nursing or subspecialties such as interventional radiology, cardiology, and critical care. Most respondents indicated their facilities had written policies regarding standard insertion and care 
TABLE 1. Participant and Facility Characteristics

\begin{tabular}{|c|c|c|}
\hline & No.* & $\%$ \\
\hline \multicolumn{3}{|l|}{ Participant characteristics } \\
\hline \multicolumn{3}{|l|}{ For how many years have you been inserting PICCs? } \\
\hline$<5$ years & 40 & $28.6 \%$ \\
\hline$\geq 5$ years & 81 & $57.9 \%$ \\
\hline \multicolumn{3}{|l|}{ Missing } \\
\hline \multicolumn{3}{|l|}{ In which of the following populations do you insert PICCs? } \\
\hline Adult patients & 121 & $86.4 \%$ \\
\hline Pediatric patients & 24 & $17.1 \%$ \\
\hline Neonatal patients & 1 & $0.7 \%$ \\
\hline \multicolumn{3}{|c|}{ In which of the following locations do you place PICCS? (Select all that apply.) } \\
\hline Adult medical ward & 115 & $82.1 \%$ \\
\hline General adult surgical ward & 110 & $78.6 \%$ \\
\hline General pediatric medical ward & 34 & $24.3 \%$ \\
\hline General pediatric surgical ward & 24 & $17.1 \%$ \\
\hline Adult intensive care unit & 114 & $81.4 \%$ \\
\hline Pediatric intensive care unit & 19 & $13.6 \%$ \\
\hline Neonatal intensive care unit & 3 & $2.1 \%$ \\
\hline Other intensive care unit & 59 & $42.1 \%$ \\
\hline Outpatient clinic or emergency department & 17 & $12.1 \%$ \\
\hline Other & 10 & $7.1 \%$ \\
\hline \multicolumn{3}{|c|}{ Approximately how many PICCS may you have placed in your career? } \\
\hline $0-99$ & 15 & $10.7 \%$ \\
\hline$\geq 100-499$ & 36 & $25.7 \%$ \\
\hline$\geq 500-999$ & 23 & $16.4 \%$ \\
\hline$\geq 1,000$ & 47 & $33.6 \%$ \\
\hline \multicolumn{3}{|l|}{ Are you the vascular access lead nurse for your facility or organization? } \\
\hline Yes & 22 & $15.7 \%$ \\
\hline No & 98 & $70.0 \%$ \\
\hline \multicolumn{3}{|c|}{ Do you currently hold a dedicated vascular access certification (BC-VA, CRNI, etc.)? } \\
\hline Yes & 32 & $22.9 \%$ \\
\hline No & 89 & $63.6 \%$ \\
\hline \multicolumn{3}{|l|}{ Facility characteristics } \\
\hline \multicolumn{3}{|l|}{ Which of the following best describes your primary work location? } \\
\hline Academic medical center & 41 & $29.3 \%$ \\
\hline For-profit community-based hospital or medical center & 30 & $21.4 \%$ \\
\hline Not-for-profit community-based hospital or medical center & 50 & $35.7 \%$ \\
\hline \multicolumn{3}{|l|}{ Who inserts the most PICCs in your facility? } \\
\hline Vascular access nurses & 133 & $95.0 \%$ \\
\hline Interventional radiology or other providers & 7 & $5.0 \%$ \\
\hline \multicolumn{3}{|l|}{ In which department is vascular access nursing located? } \\
\hline Vascular nursing & 76 & $54.3 \%$ \\
\hline General nursing & 38 & $27.1 \%$ \\
\hline Interventional radiology & 15 & $10.7 \%$ \\
\hline Other & 11 & $7.9 \%$ \\
\hline \multicolumn{3}{|c|}{ Using your best guess, how many PICCs do you think your facility inserts each month? } \\
\hline$<25$ & 5 & $3.6 \%$ \\
\hline $25-49$ & 13 & $9.3 \%$ \\
\hline $50-100$ & 39 & $27.9 \%$ \\
\hline$>100$ & 78 & $55.7 \%$ \\
\hline Unknown & 2 & $1.4 \%$ \\
\hline \multicolumn{3}{|l|}{ How many vascular access nurses are employed by your facility? } \\
\hline$<4$ & 14 & $10.0 \%$ \\
\hline $4-6$ & 33 & $23.6 \%$ \\
\hline $7-9$ & 15 & $10.7 \%$ \\
\hline $10-15$ & 25 & $17.9 \%$ \\
\hline$>15$ & 53 & $37.9 \%$ \\
\hline \multicolumn{3}{|l|}{ Does your facility track the number of PICCs placed? } \\
\hline Yes & 132 & $94.3 \%$ \\
\hline No & 5 & $3.6 \%$ \\
\hline Unknown & 3 & $2.1 \%$ \\
\hline Does your facility track the duration or dwell time of PICCs? & & \\
\hline Yes & 56 & $40.0 \%$ \\
\hline No & 60 & $42.9 \%$ \\
\hline Unknown & 24 & $17.1 \%$ \\
\hline
\end{tabular}

TABLE 1. Continued

\begin{tabular}{lcc}
\hline & No. $^{*}$ & $\%$ \\
\hline Does your facility have a written policy regarding standard PICC insertion practices? & \\
Yes & 122 & $87.1 \%$ \\
No & 8 & $5.7 \%$ \\
Unknown & 7 & $5.0 \%$ \\
Does your facility have a written policy regarding standard PICC care and maintenance? & 133 & $95.0 \%$ \\
Yes & 3 & $2.1 \%$ \\
No & 1 & $0.7 \%$ \\
Unknown & 1 \\
Does your facility have a written process to review the necessity or appropriateness of a PICC? \\
Yes & 42 & $30.0 \%$ \\
No & 63 & $45.0 \%$ \\
Unknown & 20 & $14.3 \%$ \\
\hline
\end{tabular}

NOTE: Responses may not tally to $100 \%$ for all questions due to item nonresponse. Abbreviations: BC-VA, board certified in vascular access; CRNI, certified registered nurse infusion; PICC, peripherally inserted central catheter.

practices (87\% and $95 \%$, respectively), but only $30 \%$ had policies regarding the necessity or appropriateness of PICCs.

Experience among respondents was variable: approximately a third had placed PICCs for $<5$ years $(28.6 \%)$, whereas $58 \%$ reported placing PICCs for $\geq 5$ years Correspondingly, $26 \%$ reported having placed 100 to 500 PICCs, whereas $34 \%$ had placed 1000 or more PICCs. Only $23 \%$ of those surveyed held a dedicated vascular access certification, such as board certified in vascular access or certified registered nurse infusion, whereas $16 \%$ indicated that they served as the vascular access lead nurse for their facility. Following placement, $94 \%$ of respondents reported that their facilities tracked the number of PICCs inserted, but only $40 \%$ indicated that dwell times of devices were also recorded. Only $30 \%$ of nurses reported that their hospitals had a written policy to evaluate PICC necessity or appropriateness following placement (Table 1).

The most commonly reported indications for PICC placement included intravenous antibiotics at discharge, difficult venous access, and placement for chemotherapy in patients with cancer. Forty-six percent of nurses indicated they had placed a PICC in a patient receiving some form of dialysis in the past several months; however, $91 \%$ of these respondents reported receiving approval from nephrology prior to placement in these patients. Although almost all nurses $(91 \%)$ used ultrasound to find a suitable vein for PICC placement, a smaller percentage used ultrasound to estimate the catheter-to-vein ratio to prevent thrombosis $(79 \%)$, and only a few $(14 \%)$ documented this figure in the medical record. Three-quarters of those surveyed $(76 \%)$ indicated they used ECG-based systems to position PICC tips at the cavoatrial junction to prevent thrombosis. Of those who used this technology, $36 \%$ still obtained chest $\mathrm{x}$-rays to verify the position of the PICC tip. According to $84 \%$ of respondents, flushing of PICCs was performed mainly 
TABLE 2. Practices and Care Associated With PICC Insertion and Use

\begin{tabular}{|c|c|c|}
\hline Question & No. & $\%$ \\
\hline \multicolumn{3}{|c|}{ Do you use ultrasound to find a suitable vein prior to PICC insertion? } \\
\hline Yes & 128 & $91.4 \%$ \\
\hline No & 0 & $0.0 \%$ \\
\hline \multicolumn{3}{|c|}{ Do you use ultrasound to estimate the catheter-to-vein ratio prior to PICC insertion? } \\
\hline Yes & 110 & $78.6 \%$ \\
\hline № & 18 & $12.9 \%$ \\
\hline \multicolumn{3}{|c|}{ When using ultrasound, do you document the catheter-to-vein ratio in the PICC insertion note? } \\
\hline Yes & 20 & $14.3 \%$ \\
\hline No & 89 & $63.6 \%$ \\
\hline \multicolumn{3}{|c|}{ Do you use ECG guidance-assisted systems to place PICCs? } \\
\hline Yes & 106 & $75.7 \%$ \\
\hline No & 21 & $15.0 \%$ \\
\hline \multicolumn{3}{|c|}{$\begin{array}{l}\text { If using ECG guidance, do you still routinely obtain a chest x-ray to verify PICC tip } \\
\text { position after placing the PICC using ECG guidance? }\end{array}$} \\
\hline Yes & 38 & $27.1 \%$ \\
\hline No & 68 & $48.6 \%$ \\
\hline \multicolumn{3}{|c|}{$\begin{array}{l}\text { Who is primarily responsible for administering and adhering to a flushing protocol } \\
\text { after PICC insertion at your facility? }\end{array}$} \\
\hline Bedside nurses & 118 & $83.6 \%$ \\
\hline Patients & 1 & $0.7 \%$ \\
\hline Vascular access nurses & 8 & $5.7 \%$ \\
\hline \multicolumn{3}{|c|}{ Which of the following agents are most often used to flush PICCs? } \\
\hline Both heparin and normal saline flushes & 61 & $43.6 \%$ \\
\hline Normal saline only & 63 & $45.0 \%$ \\
\hline Heparin only & 3 & $2.1 \%$ \\
\hline \multicolumn{3}{|c|}{ Who is responsible for scheduled weekly dressing changes for PICCS? } \\
\hline Vascular access nurses & 110 & $78.6 \%$ \\
\hline Bedside nurses & 14 & $10.0 \%$ \\
\hline Other (eg, IR staff, ICU staff) & 3 & $2.1 \%$ \\
\hline \multicolumn{3}{|c|}{$\begin{array}{l}\text { In the past few months, have you placed a PICC in a patient who was receiving a form of dialysis } \\
\text { (eg, peritoneal or hemodialysis)? }\end{array}$} \\
\hline Yes & 65 & $46.4 \%$ \\
\hline No & 64 & $45.7 \%$ \\
\hline \multicolumn{3}{|c|}{$\begin{array}{l}\text { If you have placed PICCs in patients on dialysis, do you discuss PICC placement or receive approva } \\
\text { from nephrology prior to inserting the PICC? }\end{array}$} \\
\hline Yes & 59 & $90.8 \%$ \\
\hline No & 6 & $9.2 \%$ \\
\hline
\end{tabular}

NOTE: Responses may not tally to $100 \%$ for all questions due to item nonresponse. Abbreviations: ECG, electrocardiography; ICU, intensive care unit; IR, interventional radiology; PICC, peripherally inserted central catheter.

by bedside nurses, whereas scheduled weekly dressing changes were most often performed by vascular access nurses (Table 2).

With respect to complications, catheter occlusion, migration, and DVT were reported as the 3 most prevalent adverse events. Interestingly, respondents did not report central line-associated bloodstream infection (CLABSI) as a common complication. Additionally, $51 \%$ of those surveyed indicated that physicians unnecessarily removed PICCs when CLABSI was suspected but not confirmed. When managing catheter occlusion, $50 \%$ of respondents began with normal saline flushes but used tissue-plasminogen activator if saline failed to resolve occlusion. Management of catheter migration varied based on degree of device movement: when the PICC had migrated $<5 \mathrm{~cm}$, most respondents $(77 \%)$ indicated they would first obtain a chest $\mathrm{x}$-ray to determine the position of the PICC tip, with few $(4 \%)$ performing catheter exchange. However, if the PICC had migrated more than $5 \mathrm{~cm}$, a significantly greater proportion of respondents $(21 \%)$ indicated they would perform a catheter exchange. With regard to managing DVT, most vascular nurses reported they notified nurses and physicians to continue using the PICC but recommended tests to confirm the diagnosis.

To better understand the experiences of vascular nurses, we asked for their perceptions regarding appropriateness of PICC use and relationships with bedside nurses, physicians, and leadership. Over a third of respondents $(36 \%)$ felt that $<5 \%$ of all PICCs may be inappropriate in their facility, whereas 1 in 5 indicated that $10 \%$ to $24 \%$ of PICCs placed in their facilities may be inappropriate or could have been avoided. Almost all $(98 \%)$ of the nurses stated they were not empowered to remove idle or clinically unnecessary PICCs without physician authorization. Although $51 \%$ of nurses described the support received from hospital leadership as excellent, very good, or good, $43 \%$ described leadership support as either fair or poor. Conversely, relationships with bedside nurses and physicians were rated as being very good or good by nearly two-thirds of those surveyed (64\% and $65 \%$, respectively) (Table 3 ).

\section{Variation in Responses Based on Years in Practice or Certification}

We initially hypothesized that responses regarding practice (ultrasound use, ECG guidance system use), management of complications, or perceptions regarding leadership might vary based on years of experience, number of PICCs placed, or certification status. However, no statistically significant associations with these factors and individual responses were identified.

\section{DISCUSSION}

In this survey of 140 vascular access nurses in hospitals across Michigan, new insights regarding the experience, practice, knowledge, and beliefs of this group of providers were obtained. We found that vascular access nurses varied with respect to years in practice, volume of PICCs placed, and certification status, reflecting heterogeneity in this provider group. Variation in insertion techniques, such as use of ultrasound to examine catheter-to-vein ratio (a key way to prevent thrombosis) or newer ECG technology to position the PICC, was also noted. Although indications for PICC insertion appeared consistent with published literature, the frequency with which these devices were placed in patients receiving dialysis (reportedly with nephrology approval) was surprising given national calls to avoid such use. ${ }^{16}$ Opportunities to improve hospital practices, such as tracking PICC dwell times and PICC necessity, as well as the potential need to better educate physicians on when to remove PICCs for suspected CLABSI, were also identified. Collectively, these data are highly relevant to 
TABLE 3. Approach to PICC-Associated Complications, Relationships, and Empowerment

\begin{tabular}{|c|c|c|}
\hline Question & No. & $\%$ \\
\hline \multicolumn{3}{|l|}{ Which of the following PICC-related complications have you most frequently encountered in your practice? } \\
\hline Catheter occlusion & 81 & $57.9 \%$ \\
\hline Catheter migration & 27 & $19.3 \%$ \\
\hline PICC-associated DVT & 6 & $4.3 \%$ \\
\hline Catheter fracture or embolization & 3 & $2.1 \%$ \\
\hline Exit site infection & 3 & $2.1 \%$ \\
\hline Coiling or kinking after insertion & 2 & $1.4 \%$ \\
\hline \multicolumn{3}{|c|}{ If you suspect a patient has catheter occlusion, which of the following best describes your approach to resolving this problem? } \\
\hline Begin with normal saline but use a tPA product if this fails to restore patency & 70 & $50.0 \%$ \\
\hline Use a tPA product (eg, Cathflo, Activase, or Retavase) to restore patency & 44 & $31.4 \%$ \\
\hline Begin with heparin-based flushes but use a tPA product if this fails to restore & 7 & $5.0 \%$ \\
\hline Use only normal saline flushes to restore patency & 3 & $2.1 \%$ \\
\hline \multicolumn{3}{|c|}{ If you find a PICC that has migrated out or has been accidentally dislodged $<5 \mathrm{~cm}$ in a patient without symptoms, and the device is still clinically needed, which of the following best describes your practice? } \\
\hline Obtain a chest $x$-ray to verify tip position & 108 & $77.1 \%$ \\
\hline Perform a complete catheter exchange over a guidewire if possible & 5 & $3.6 \%$ \\
\hline Notify/discuss next steps with physician & 5 & $3.6 \%$ \\
\hline Other & 6 & $4.3 \%$ \\
\hline \multicolumn{3}{|c|}{ If you find a PICC that has migrated out or has been accidentally dislodged $>5 \mathrm{~cm}$ in a patient without symptoms, and the device is still clinically needed, which of the following best describes your practice? } \\
\hline Obtain a chest $x$-ray to verify tip position & 72 & $51.4 \%$ \\
\hline Perform a catheter exchange over a guidewire if possible & 30 & $21.4 \%$ \\
\hline Notify/discuss next steps with physician & 10 & $7.1 \%$ \\
\hline Other & 12 & $8.6 \%$ \\
\hline \multicolumn{3}{|l|}{ Which of the following best describes your first approach when you suspect a patient has PICC-associated phlebitis? } \\
\hline Discuss best course of action with physician or nurse & 79 & $56.4 \%$ \\
\hline Supportive measures (eg, warm compresses, analgesics, monitoring) & 25 & $17.9 \%$ \\
\hline Remove the PICC & 15 & $10.7 \%$ \\
\hline Other & 5 & $3.6 \%$ \\
\hline \multicolumn{3}{|l|}{ Which of the following best describes your first approach when you suspect a patient has a PICC-related DVT? } \\
\hline Notify caregivers to continue using PICC and consider tests such as ultrasound & 82 & $58.6 \%$ \\
\hline Notify bedside nurse and physician not to continue use of the PICC and consider tests such as ultrasound & 42 & $30.0 \%$ \\
\hline \multicolumn{3}{|c|}{ PICCS are often removed when physicians suspect, but have not yet confirmed, CLABSI. Considering your experiences, what percentage of PICCs may have been removed in this manner at your facility? } \\
\hline$<5 \%$ & 11 & $7.9 \%$ \\
\hline $5-9 \%$ & 16 & $11.4 \%$ \\
\hline $10-24 \%$ & 24 & $17.1 \%$ \\
\hline$\geq 25 \%$ & 71 & $50.7 \%$ \\
\hline \multicolumn{3}{|c|}{ Based on your experience, what percentage of PICCs do you think are inappropriate or could have been avoided at your facility? } \\
\hline$<5 \%$ & 51 & $36.4 \%$ \\
\hline $5-9 \%$ & 25 & $17.9 \%$ \\
\hline $10-24 \%$ & 28 & $20.0 \%$ \\
\hline $25-50 \%$ & 13 & $9.3 \%$ \\
\hline$>50 \%$ & 5 & $3.6 \%$ \\
\hline \multicolumn{3}{|c|}{ Are vascular access nurses empowered to remove PICCs that are idle or clinically unnecessary without physician authorization? } \\
\hline Yes & 3 & $2.1 \%$ \\
\hline No & 122 & $87.1 \%$ \\
\hline \multicolumn{3}{|l|}{ How would you rank the overall support your vascular access service receives from hospital leadership? } \\
\hline Excellent & 5 & $3.6 \%$ \\
\hline Very good & 32 & $22.9 \%$ \\
\hline Good & 40 & $28.6 \%$ \\
\hline Fair & 35 & $25.0 \%$ \\
\hline Poor & 25 & $17.9 \%$ \\
\hline \multicolumn{3}{|c|}{ How would you describe your relationship with physicians at your facility when it comes to communicating recommendations or management of PICCS? } \\
\hline Very good & 28 & $20.0 \%$ \\
\hline Good & 63 & $45.0 \%$ \\
\hline Fair & 35 & $25.0 \%$ \\
\hline Poor & 7 & $5.0 \%$ \\
\hline Very poor & 4 & $2.9 \%$ \\
\hline \multicolumn{3}{|c|}{ How would you describe your relationship with bedside nurses at your facility when it comes to communicating recommendations or management of PICCS? } \\
\hline Very good & 32 & $22.9 \%$ \\
\hline Good & 58 & $41.4 \%$ \\
\hline Fair & 38 & $27.1 \%$ \\
\hline Poor & 7 & $5.0 \%$ \\
\hline Very poor & 2 & $1.4 \%$ \\
\hline
\end{tabular}

NOTE: Responses may not tally to $100 \%$ for all questions due to item nonresponse. Abbreviations: CLABSI, central line-associated bloodstream infection; DVT, deep vein thrombosis; PICC, peripherally inserted central catheter; tPA, tissue plasminogen activator. 
hospitalists and health systems as they help to identify areas for quality improvement and inform clinical practice regarding the use of PICCs in hospitalized patients. As hospitalists increasingly order PICCs and manage complications associated with these devices, they are well suited to use these data so as to improve patient safety and clinical outcomes.

Venous access is the most common medical procedure performed in hospitalized medical patients. Although a number of devices including peripheral intravenous catheters, central venous catheters, and PICCs are used for this purpose, the growing use of PICCs to secure venous access has been documented in several studies. ${ }^{17}$ Such growth, in part, undoubtedly reflects increasing availability of vascular access nurses. Traditionally placed by interventional radiologists, the creation of dedicated vascular nursing teams has resulted in these subspecialists now serving in more of a "backup" or "trouble-shooting" role rather than that of primary operator. ${ }^{4,14}$ This paradigm shift is well illustrated in a recent survey of infection preventionists, where over $60 \%$ of respondents reported that they had a vascular nursing team in their facility. ${ }^{7}$ The growth of these nursing-led vascular access teams has produced not only high rates of insertion success and low rates of complications, but also greater cost-effectiveness when compared to interventional radiology-based insertion. ${ }^{18}$

Nonetheless, our survey also identified a number of important concerns regarding PICC practices and vascular nursing providers. First, we found variation in areas such as insertion practices and management of complications. Such variability highlights the importance of both growing and disseminating the evidence base for consistent practice in vascular nursing. Through their close clinical affiliation with vascular nurses and shared interests in obtaining safe and appropriate venous access for patients, hospitalists are ideally poised to lead this effort. Second, similarities between vascular nurse opinions regarding appropriateness of PICCs and those of hospitalists from a prior survey were noted. ${ }^{19}$ Namely, a substantial proportion of both vascular nurses and hospitalists felt that some PICCs were inappropriate and could be avoided. Third, although relationships between vascular access nurses and leadership were reported as being variable, the survey responses suggested relatively good interprovider relationships with bedside nurses and physicians. Such relationships likely reflect the close clinical ties that emerge from "being in the trenches" of patient care and suggest that interventions to improve care in partnership with these providers are highly viable.

Our study has some limitations. First, despite a high response rate, our study used a survey design and reports findings from a convenience sample of vascular access nurses in a single state. Thus, nonrespondent and selection biases remain threats to our conclusions. Additionally, some respondents did not complete all responses, perhaps due to nonapplicability to practice or other unknown reasons. The pattern of missingness observed, however, suggested that such responses were missing at random. Second, we surveyed vascular nurses in hospitals that are actively engaged in improving PICC practices; our findings may therefore not be representative of vascular nursing professionals as a whole and may instead reflect those of a highly motivated group of individuals. Relatedly, the underlying reasons for adoption of specific practices or techniques cannot be discerned from our study. Third, although we did not find differences based on years in practice or certification status, our sample size was relatively small and likely underpowered for these comparisons. Finally, our study sample consists of vascular nurses who are clustered within hospitals in which they are employed. Therefore, overlap between reported practices and those required by the facility are possible.

Despite these limitations, our study has important strengths. First, this is among the most comprehensive of surveys examining vascular nursing experience, practice, knowledge, and beliefs. The growing presence of these providers across US hospitals, coupled with limited insight regarding their clinical practices, highlight the importance and utility of these data. Second, we noted important differences in experience, practices, and interprovider relationships between vascular providers in this field. Although we are unable to ascertain the drivers or significance of such variation, hospitals and health systems focused on improving patient safety should consider quantifying and exploring these factors. Third, findings from our survey within Michigan suggest the need for similar, larger studies across the country. Partnerships with nursing organizations or larger professional groups that represent vascular nursing specialists may be helpful in this regard.

In conclusion, we found important similarities and differences in vascular nursing experience, practice, knowledge, and beliefs in Michigan. These data are useful as they help provide context regarding the constitution of these teams, current practices, and opportunities for improving care. Hospitalists seeking to improve patient safety may use these data to better inform vascular access practice in hospitalized patients.

\footnotetext{
Acknowledgements

The authors thank Claire Rickard, PhD, RN, Britt Meyer, RN, Peter Carr, PhD, and David Dempsey, RN for their assistance in developing the survey instrument used in this study.

Disclosures: This project was funded through an Investigator Initiated Research Grant from the Blue Cross Blue Shield of Michigan (BCBSM) Foundation (grant number 2140.II). The funding source played no role in study design, data acquisition, analysis, or reporting of the data. Support for the Hospital Medicine Safety (HMS) Consortium is provided by BCBSM and the Blue Care Network as part of the BCBSM Value Partnerships program. Although BCBSM and HMS work collaboratively, the opinions, beliefs, and viewpoints expressed by the authors do not necessarily reflect the opinions, beliefs, and viewpoints of BCBSM or any of its employees. This work was also supported with resources from the Veterans Affairs Ann Arbor Healthcare System. The views expressed in this article are those of the authors and do not necessarily reflect the position or policy of the Department of Veterans Affairs or the United States government.
} 


\section{References}

1. Al Raiy B, Fakih MG, Bryan-Nomides N, et al. Peripherally inserted central venous catheters in the acute care setting: a safe alternative to high-risk short-term central venous catheters. Am J Infect Control. 2010;38(2):149-153.

2. Lobo BL, Vaidean G, Broyles J, Reaves AB, Shorr RI. Risk of venous thromboembolism in hospitalized patients with peripherally inserted central catheters. J Hosp Med. 2009;4(7):417-422.

3. Alexandrou E, Spencer T, Frost S, Mifflin N, Davidson P, Hillman K. Central venous catheter placement by advanced practice nurses demonstrates low procedural complication and infection rates--a report from 13 years of service. Crit Care Med. 2014;42(3):536-543.

4. Meyer B. Developing an alternative workflow model for peripherally inserted central catheter placement. J Infus Nurs. 2012;34(1): 34-42.

5. Burns T, Lamberth B. Facility wide benefits of radiology vascular access teams. Radiol Manage. 2010;32(1):28-32; quiz 33-34.

6. Meyer BM, Chopra V. Moving the needle forward: the imperative for collaboration in vascular access. J Infus Nurs. 2015;38(2):100-102.

7. Krein S, Kuhn L, Ratz D, Chopra V. Use of designated PICC teams by U.S. hospitals: a survey-based study [published online November 10, 2015]. J Patient Saf. doi: 10.1097/PTS.0000000000000246

8. Greene MT, Flanders SA, Woller SC, Bernstein SJ, Chopra V. The association between PICC use and venous thromboembolism in upper and lower extremities. American J Med. 2015;128(9):986-993.e1.

9. Flanders SA, Greene MT, Grant P, et al. Hospital performance for pharmacologic venous thromboembolism prophylaxis and rate of venous thromboembolism: a cohort study. JAMA Intern Med. 2014; 174(10):1577-1584
10. Chopra V, Anand S, Hickner A, et al. Risk of venous thromboembolism associated with peripherally inserted central catheters: a systematic review and meta-analysis. Lancet. 2013;382(9889):311-325.

11. Infusion Nurses Society. Infusion nursing standards of practice. J Infus Nurs. 2006;29(1 suppl):S1-S92.

12. O'Grady NP, Alexander M, Burns LA, et al. Guidelines for the prevention of intravascular catheter-related infections. Am J Infect Control. 2011;39(4 suppl 1):S1-S34.

13. Lamperti M, Bodenham AR, Pittiruti M, et al. International evidencebased recommendations on ultrasound-guided vascular access. Intensive Care Med. 2012;38(7):1105-1117.

14. Sainathan S, Hempstead M, Andaz S. A single institution experience of seven hundred consecutively placed peripherally inserted central venous catheters. J Vasc Access. 2014;15(6):498-502.

15. Broadhurst D, Moureau N, Ullman AJ. Central venous access devices site care practices: an international survey of 34 countries [published online September 3, 2015]. J Vasc Access. doi: 10.5301/jva.5000450

16. American Society of Nephrology. World's Leading Kidney Society Joins Effort to Reduce Unnecessary Medical Tests and Procedures. Available at: https://www.asn-online.org/policy/choosingwisely/PressReleaseChoosingWisely.pdf. Accessed September 4, 2015.

17. Johansson E, Hammarskjold F, Lundberg D, Heibert Arnlind M. A survey of the current use of peripherally inserted central venous catheter (PICC) in Swedish oncology departments. Acta Oncol. 2013;52(6):1241-1242.

18. Walker G, Todd A. Nurse-led PICC insertion: is it cost effective? Br J Nurs. 2013;22(19):S9-S15.

19. Chopra V, Kuhn L, Coffey CE Jr, et al. Hospitalist experiences, practice, opinions, and knowledge regarding peripherally inserted central catheters: a Michigan survey. J Hosp Med. 2013;8(6):309-314. 